\title{
Intravitreal Aflibercept Versus Photodynamic Therapy in Chinese Patients with Neovascular Age-Related Macular Degeneration: Outcomes of the SIGHT Study
}

\author{
Xiaoxin Li, ${ }^{1}$ Youxin Chen, Junjun Zhang, ${ }^{3}$ Xun Xu, Feng Zhang, Chui Ming Gemmy Cheung, ${ }^{6}$ Rui Yu, \\ Husain Kazmi, ${ }^{8}$ Olaf Sowade, Oliver Zeitz'; on behalf of the SIGHT study group ${ }^{*}$
}

\begin{abstract}
Purpose: SIGHT compared intravitreal aflibercept injections (IAI) with photodynamic therapy (PDT) in Chinese patients with predominantly classic choroidal neovascularization $(\mathrm{CNV})$ secondary to neovascular agerelated macular degeneration (nAMD).

Methods: Patients were randomized 3:1 to IAI ( $2 \mathrm{mg}$ every 8 weeks after 3 initial monthly injections)/sham PDT or active PDT/sham IAI (with switch to IAI at week 28). We report the primary outcome (mean change in best-corrected visual acuity [BCVA] at week 28) and final 52-week outcomes.

Results: Patients were randomized to IAI $(n=228)$ or PDT $(n=76)$ (mean age: 65.1 years). Mean BCVA change was +14.0 (IAI) versus +3.9 letters (PDT $\rightarrow$ IAI) (week 28 ) and +15.2 versus +8.9 letters (between-group difference: 6.2 letters; $P=0.0009)$ (week 52); mean reduction in central retinal thickness was -189.6 versus $-170.0 \mu \mathrm{m}$ (week 52). The greatest improvements in BCVA with IAI were in youngest patients ( $<65$ years), and in those with a smaller active component of the CNV lesion ( $<50 \%$ of lesion size). The most common ocular treatment-emergent adverse events (study eye; IAI vs. PDT $\rightarrow$ IAI) were macular fibrosis $(11.8 \%$ vs. $6.6 \%)$ and visual acuity reduced (6.6\% vs. $21.1 \%$ ). Three treatment-emergent Antiplatelet Trialists' Collaboration-defined arterial thromboembolic events were observed but none was considered drug related.

Conclusions: IAI demonstrated superiority over PDT in Chinese nAMD patients. The benefits of IAI were maintained through week 52 in all patients, including subgroups, and in patients who switched from PDT to IAI. The incidence of adverse events was consistent with the known safety profile of IAI. Trial registration number NCT01482910.
\end{abstract}

Keywords: intravitreal aflibercept, SIGHT, neovascular age-related macular degeneration, photodynamic therapy

\footnotetext{
${ }^{1}$ Department of Ophthalmology, People's Eye Center, Peking University People's Hospital, Xicheng District, Beijing, China.

${ }^{2}$ Department of Ophthalmology, Peking Union Medical Colleague Hospital, Dongcheng District, Beijing, China.

${ }^{3}$ Department of Ophthalmology, West China School of Medicine/West China Hospital, Sichuan University, Wuhou District, Chengdu, China.

${ }^{4}$ Department of Ophthalmology, Shanghai General Hospital, Hongkou District, Shanghai, China.

${ }^{5}$ Department of Ophthalmology, Beijing Tongren Hospital, CMU, Dongcheng District, Beijing, China.

${ }^{6}$ Department of Ophthalmology, Singapore Eye Research Institute, Singapore, Singapore.

${ }^{7}$ Department of Ophthalmology, Bayer US, Whippany, New York.

${ }^{8}$ Ophthalmology, Regeneron Pharmaceuticals, Inc., Tarrytown, New York.

${ }^{9}$ Development, Pharmaceuticals, Bayer AG, Berlin, Germany.

*Members of the SIGHT study group in China.

(C) Xiaoxin Li, et al., 2017; Published by Mary Ann Liebert, Inc. This is an Open Access article distributed under the terms of the Creative Commons Attribution License, which permits unrestricted use, distribution, and reproduction in any medium, provided the original work is properly cited.
} 


\section{Introduction}

T IS CURREnTLy estimated that around 170 million people globally, including 59 million people in Asia, have agerelated macular degeneration (AMD). These numbers are predicted to increase to 288 million globally with 113 million in Asia by 2040. ${ }^{1}$ The sizeable growth predicted in Asia underscores the need for therapies validated in patients originating from this region. In China and other Asian countries, photodynamic therapy (PDT) is a well-established treatment of choroidal neovascularization (CNV) in neovascular AMD (nAMD). ${ }^{2-4}$ Anecdotal evidence shows good efficacy of PDT in many Asian patients, which on average seems better than the PDT results observed in Western populations. ${ }^{2,4-6}$ However, studies comparing PDT with therapies targeting vascular endothelial growth factor pathways, the current global standard of care, have predominantly been in Western populations. ${ }^{2,3}$ Evidence in an Asian, and specifically a Chinese, population is limited. Therefore, a head-to-head comparison of intravitreal aflibercept injections (IAI) versus PDT is warranted for scientific reasons to confirm superiority versus PDT as the previous gold standard in this population.

IAI is widely used for the treatment of nAMD. The efficacy and safety of IAI have been established by the global VIEW studies, in which patients with nAMD were randomized to IAI ( 0.5 or $2 \mathrm{mg}$ every 4 weeks or $2 \mathrm{mg}$ every 8 weeks [2q8] after 3 initial monthly injections) or intravitreal ranibizumab $0.5 \mathrm{mg}$ every 4 weeks. ${ }^{7,8}$ IAI in all treatment groups was clinically equivalent and noninferior to intravitreal ranibizumab for the primary outcome (vision maintenance at week 52), and the incidence of ocular serious adverse events (AEs) was comparable between the IAI $2 \mathrm{q} 8(2.0 \%)$ and intravitreal ranibizumab $(3.2 \%)$ groups at week 52. ${ }^{7,8}$ Eleven percent of patients in the VIEW studies were Asian (most were Japanese), but subgroup analysis showed that IAI was similarly effective to ranibizumab in these patients. ${ }^{9}$ Chinese patients were not enrolled in VIEW.

The primary objective of the SIGHT study was to assess the efficacy and safety of IAI compared with PDT in Chinese patients with predominantly classic CNV secondary to nAMD. PDT was used according to label, meaning that the study was focused on predominantly classic CNV and retreatments were allowed. SIGHT also compared the effects of treatment in prespecified subgroups based on age and size of active CNV lesions as previous reports suggest that lesion size and age might be predictive for the success of PDT. ${ }^{10}$ In this article, we report the primary outcome from SIGHT (mean change in best-corrected visual acuity [BCVA] at week 28), and the final 52-week efficacy and safety outcomes in all patients, including subgroups.

\section{Methods}

\section{Study design}

SIGHT (NCT01482910) was a 52-week, phase 3, randomized, double-masked, multicenter study conducted from December 2011 to August 2014 in 14 sites across China. SIGHT evaluated the efficacy and safety of IAI versus PDT in patients with predominantly classic CNV secondary to nAMD. SIGHT was performed in accordance with the International Conference on Harmonisation Good Clinical
Practice guidelines and the Declaration of Helsinki. The protocol and amendments were approved by the independent ethics committees and institutional review boards for all participating centers in China. All patients provided written informed consent to participate.

\section{Participants}

Adults aged $\geq 50$ years with active, predominantly classic subfoveal CNV secondary to nAMD in the study eye were eligible. Patients were also required to have BCVA between 73 and 25 Early Treatment Diabetic Retinopathy Study (ETDRS) letters (Snellen activity equivalent of 20/40 to 20/320) in the study eye, and be able to comply with the requirements of the protocol in the view of the investigator. Eligibility was based on medical history, ophthalmic and physical examinations, laboratory test results, optical coherence tomography (OCT), indocyanine green angiography (ICGA), fundus photography, and fluorescein angiography (FA) before randomization during a screening period. Eligibility, based on fundus photography, FA, and ICGA results, was confirmed by a Central Reader Center. Polypoidal choroidal vasculopathy (PCV) was excluded by ICGA and/or fundoscopic images/FA and medical records. Complete exclusion criteria are summarized in Supplementary Table S1 (Supplementary materials are available online at www.liebertpub.com/jop).

\section{Randomization and treatments}

Treatment was allocated to patients using a computergenerated randomization list. Eligible patients were randomized in a 3:1 ratio to IAI plus sham PDT or active PDT plus sham injection. Patients in the IAI group received $2 \mathrm{mg}$ injections at baseline, weeks 4, 8, 16, 24, 32, 40, and 48, including sham injections at weeks 28 and 36 (to maintain masking). These patients also received a sham PDT procedure at baseline and further sham procedures at weeks 12 and 24, if they met the same retreatment criteria that were used in the PDT group. Patients in the PDT group received one mandatory active PDT procedure at baseline, with those meeting the criteria for PDT retreatment receiving further treatments at weeks 12 and 24. The criteria for PDT retreatment were based on the clinical judgment of the masked investigator in accordance with published guidelines ${ }^{11}$ and the verteporfin package insert. Patients in the PDT group also received a sham injection at baseline and at weeks 4,8 , 16 , and 24, followed by IAI $2 \mathrm{mg}$ injections at weeks 28, 32, 36, 40, and 48 (PDT $\rightarrow$ IAI) (Supplementary Fig. S1).

No approved or investigational agents for the treatment of nAMD were permitted in the study eye until early termination or final assessments. All study treatments (active and sham) were administered by unmasked personnel. All other study site personnel remained masked to the treatment assignment of all patients through to week 52. All drugs were supplied by Bayer Pharmaceuticals (Leverkusen, Germany).

\section{Outcome measures}

The primary efficacy outcome was the mean change in BCVA (ETDRS letters) from baseline to week 28. Standardization of BCVA measurements throughout sites and countries was ensured by a standardized face-to-face training and certification through a specific vendor (EMMES), 
using certified and standardized equipment and procedures. The confirmatory secondary endpoint was the proportion of patients who lost $<15$ ETDRS letters at week 28. The other secondary endpoints (exploratory) at weeks 28 and 52 were the proportion of patients who gained or lost $\geq 5, \geq 10$, or $\geq 15$ ETDRS letters; change from baseline in central retinal thickness (CRT) assessed via OCT; change from baseline in $\mathrm{CNV}$ lesion size assessed by FA; and changes from baseline in EuroQoL-5D (EQ-5D) and National Eye Institute Visual Function Questionnaire-25 item (NEI VFQ-25) scores.

Safety assessments included the proportion of patients with treatment-emergent AEs (TEAEs). An AE was considered treatment emergent if it was observed or reported after the first and not later than 30 days after last administration of study drug. All AEs were coded using Medical Dictionary for Regulatory Activities (MedDRA) version 17.0. A masked Antiplatelet Trialists' Collaboration (APTC) Adjudication Committee (two sponsor cardiovascular medicine experts and two external cardiologists) classified all potential treatment-emergent vascular events based on the APTC classification for arterial thromboembolic events (ATEs).

Patients were examined at screening, baseline, week 1, week 4 , and every 4 weeks thereafter to week 52 . Every visit included assessment of BCVA, OCT, and vital signs, and slit lamp (including intraocular pressure measurement) before and 30-60 min after active or sham injection. Patients completed the EQ-5D and NEI VFQ-25 questionnaires at baseline and weeks 28 and 52. Fundus photography and FA were performed at screening, and at weeks $12,24,28$, and 52. Any information on AEs, including intensity, causal relationship to study drug, action taken, and outcome, was recorded in electronic case report forms during clinic visits. The presence of anti-IAI antibodies was determined (using serum samples) before the first dose at baseline and again before treatment at weeks 16,28 , and 52 .

\section{Statistical analyses}

A total of 300 patients were required to ensure $90 \%$ power to detect treatment difference (superiority) assuming a treatment difference of at least 7.5 ETDRS letters. This estimation was based on a randomization ratio of 3:1, a two-sided significance level of 5\%, and a common standard deviation (SD) of 14 letters. The basis for this SD was the observed SD of the IAI 2 q8 arm of the VIEW 2 study. ${ }^{7}$ All randomized patients who received any study drug and had baseline and $\geq 1$ postbaseline BCVA assessment were included in the full analysis set. All randomized patients who received any study drug were included in the safety analysis set.

The primary endpoint was evaluated in the full analysis set using an analysis of covariance (ANCOVA) with baseline BCVA as a covariate, and treatment group and baseline BCVA group ( $<45$ letters vs. $\geq 45$ letters) as fixed factors. If superiority was shown on the primary endpoint, an additional comparison of superiority was tested with respect to the confirmatory secondary endpoint. This endpoint was analyzed using the Cochrane-Mantel-Haenszel (CMH) test, adjusted for baseline BCVA group ( $<45$ letters vs. $\geq 45$ letters).

All other secondary analyses, including those performed in prespecified subgroups (sex, age, baseline CNV lesion size, and baseline BCVA), were exploratory with descriptive $P$-values. An ANCOVA method was used to analyze continuous variables (such as BCVA and CRT). The least squares mean (LSM) and the two-sided 95\% confidence interval (CI) of the comparator-subtracted treatment difference were calculated from the ANCOVA models. Binary outcomes were analyzed using a $\mathrm{CMH}$ method. A point estimate and a two-sided 95\% CI using normal approximation for the treatment difference of IAI minus PDT were calculated using the $\mathrm{CMH}$ tests. For both primary and confirmatory secondary endpoints, IAI was considered superior to PDT if the CI of the comparator-subtracted difference was above 0 , where a positive difference favors IAI. A last observation carried forward (LOCF) approach was applied to impute missing values. All safety assessments were analyzed descriptively. Statistical analyses were performed using SAS 9.2 (SAS Institute, Inc., Cary, NC).

\section{Results}

\section{Patient characteristics, disposition, and treatment exposure}

A total of 304 patients were randomized to treatment with IAI $(n=228)$ or PDT $\rightarrow$ IAI $(n=76)$. All 304 patients were included in both the efficacy and safety analyses. Baseline characteristics were comparable between treatment groups (Table 1). Most patients were male (64.5\%), the mean age was 65.1 years, and the mean baseline BCVA was 49.8 letters.

The majority of randomized patients in the IAI (94.7\%) and PDT $\rightarrow$ IAI groups (93.4\%) completed 28 weeks of treatment and entered the second half of the study (i.e., from weeks 28 to 52), and patients in both groups received IAI during this period. A total of 17 patients discontinued before week 28 (IAI, $n=12$; PDT $\rightarrow$ IAI, $n=5$ ), and 7 patients (IAI, $n=3$; PDT $\rightarrow$ IAI, $n=4$ ) discontinued treatment between weeks 28 and 48 (Fig. 1). The most common reason for discontinuation was "adverse event," for which the rate of discontinuation due to $\mathrm{AE}$ was lower in the IAI group $(2.6 \% ; n=6 / 228)$ than the PDT $\rightarrow$ IAI group $(6.6 \% ; n=5 /$ 76). The reasons for withdrawal due to TEAE included macular degeneration $(n=1)$, visual acuity reduced $(n=1)$, pathologic fracture $(n=1)$, and plasmacytoma $(n=1)$ in the IAI group, and acute myocardial infarction $(n=1)$, angina pectoris $(n=1)$, visual acuity reduced $(n=2)$, and cerebral infarction $(n=1)$ in the PDT $\rightarrow$ IAI group.

Over the course of the 52-week study, 93.0\% $(n=212 /$ 228) of patients in the IAI group received a maximum of eight active IAI $2 \mathrm{mg}$ injections, and $86.8 \%(n=66 / 76)$ of patients in the PDT $\rightarrow$ IAI received a maximum of five active IAI $2 \mathrm{mg}$ injections (from week 28 onward). The mean number of active PDT treatments was 0 in the IAI group and 1.8 in the PDT $\rightarrow$ IAI group up to week 28 . There were no further active PDT retreatments from weeks 28 to 52 .

\section{Functional outcomes}

IAI was statistically superior to PDT for the primary and confirmatory secondary endpoints. The mean change in BCVA letter score (primary outcome) from baseline to week 28 was +14.0 letters in the IAI group compared with +3.9 letters in the PDT $\rightarrow$ IAI group. The improvement in BCVA was maintained at week 52 in the IAI group (+15.2 letters) and improved after switch to IAI at week 28 in the PDT $\rightarrow$ IAI group. In this group, the difference from weeks 28 to 52 was +5.4 letters, with a final mean change of +8.9 letters from baseline to week 52 (Fig. 2). The LSM between-group 
Table 1. Baseline Characteristics of the Randomized Population

\begin{tabular}{|c|c|c|c|}
\hline Characteristics & $I A I(\mathrm{n}=228)$ & $P D T(\mathrm{n}=76)$ & Total $(\mathrm{n}=304)$ \\
\hline Asian, $n(\%)$ & $228(100.0)$ & $76(100.0)$ & $304(100.0)$ \\
\hline Male, $n(\%)$ & $142(62.3)$ & $54(71.1)$ & $196(64.5)$ \\
\hline Left study eye, $n(\%)$ & $122(53.5)$ & $38(50.0)$ & $160(52.6)$ \\
\hline Age (years), mean \pm SD & $65.1 \pm 8.8$ & $64.9 \pm 8.5$ & $65.1 \pm 8.7$ \\
\hline$<65$ years, $n(\%)$ & $111(48.7)$ & $36(47.4)$ & $147(48.4)$ \\
\hline$\geq 65$ to $<75$ years, $n(\%)$ & $78(34.2)$ & $31(40.8)$ & $109(35.9)$ \\
\hline$\geq 75$ years, $n(\%)$ & $39(17.1)$ & $9(11.8)$ & $48(15.8)$ \\
\hline \multicolumn{4}{|l|}{ CNV lesions, $n(\%)$} \\
\hline Classic & $216(94.7)$ & $73(96.1)$ & $289(95.1)$ \\
\hline Classic and occult & $6(2.6)$ & $3(3.9)$ & $9(3.0)$ \\
\hline Occult & $3(1.3)$ & $0(0.0)$ & $3(1.0)$ \\
\hline Missing & $3(1.3)$ & $0(0.0)$ & $3(1.0)$ \\
\hline Total lesion size $\left(\mathrm{mm}^{2}\right)$, mean $\pm \mathrm{SD}(n=301)$ & $3.274 \pm 3.848$ & $3.207 \pm 3.836$ & $3.257 \pm 3.839$ \\
\hline CNV lesion size $\left(\mathrm{mm}^{2}\right)$, mean $\pm \mathrm{SD}(n=301)$ & $1.812 \pm 2.350$ & $1.884 \pm 2.459$ & $1.830 \pm 2.374$ \\
\hline $\mathrm{CNV}<50 \%$ of total lesion & $78(34.2)$ & $28(36.8)$ & $106(34.9)$ \\
\hline $\mathrm{CNV} \geq 50 \%$ of total lesion & $147(64.5)$ & $48(63.2)$ & $195(64.1)$ \\
\hline Missing & $3(1.3)$ & $0(0.0)$ & $3(1.0)$ \\
\hline Area of leakage $\left(\mathrm{mm}^{2}\right)$, mean $\pm \mathrm{SD}(n=298)$ & $3.621 \pm 3.823$ & $3.557 \pm 4.035$ & $3.605 \pm 3.872$ \\
\hline BCVA ETDRS letters, mean \pm SD & $49.9 \pm 13.4$ & $49.6 \pm 13.8$ & $49.8 \pm 13.5$ \\
\hline \multicolumn{4}{|l|}{ BCVA ETDRS letter score, $n(\%)$} \\
\hline$<45$ letters & $76(33.3)$ & $27(35.5)$ & $103(33.9)$ \\
\hline$\geq 45$ letters & $152(66.7)$ & $49(64.5)$ & $201(66.1)$ \\
\hline $\mathrm{CRT}(\mu \mathrm{M})$, mean $\pm \mathrm{SD}$ & $457.5 \pm 169.5$ & $467.4 \pm 175.2$ & $460.0 \pm 170.7$ \\
\hline NEI VFQ-25 total score, mean \pm SD $(n=303)$ & $74.11 \pm 15.61$ & $73.87 \pm 16.05$ & $74.05 \pm 15.70$ \\
\hline EQ-5D total score, mean \pm SD $(n=303)$ & $0.93 \pm 0.11$ & $0.95 \pm 0.09$ & $0.94 \pm 0.10$ \\
\hline
\end{tabular}

BCVA, best-corrected visual acuity; CNV, choroidal neovascularization; CRT, central retinal thickness; EQ-5D, EuroQoL-5D; ETDRS, Early Treatment Diabetic Retinopathy Study; IAI, intravitreal aflibercept injections; NEI VFQ-25, National Eye Institute Visual Function Questionnaire-25 item; PDT, photodynamic therapy; SD, standard deviation.

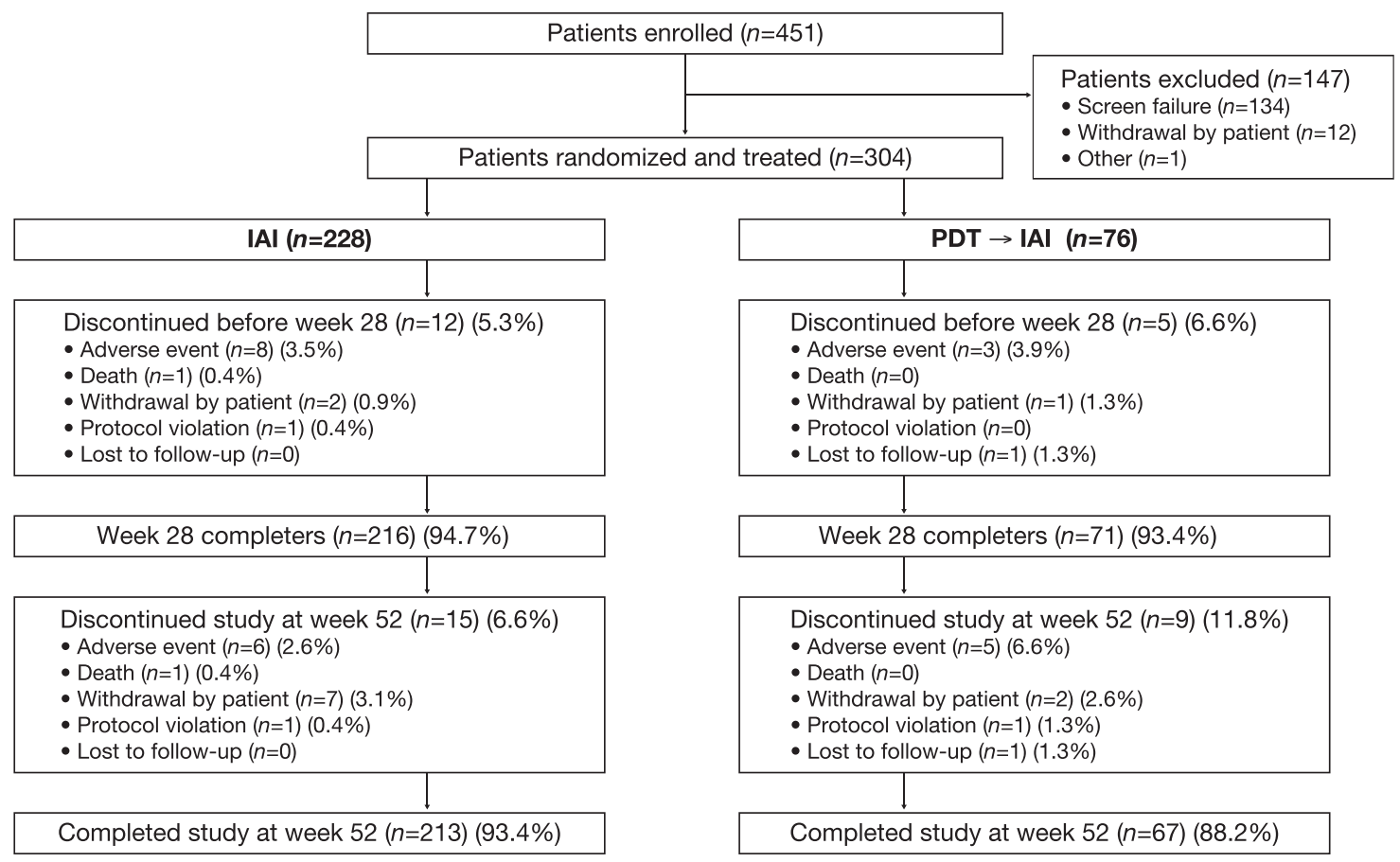

FIG. 1. Patient disposition during the 52-week study. Patients in the PDT group switched to IAI at week 28 (after the primary efficacy assessment) (PDT $\rightarrow$ IAI). IAI, intravitreal aflibercept injections; PDT, photodynamic therapy. 


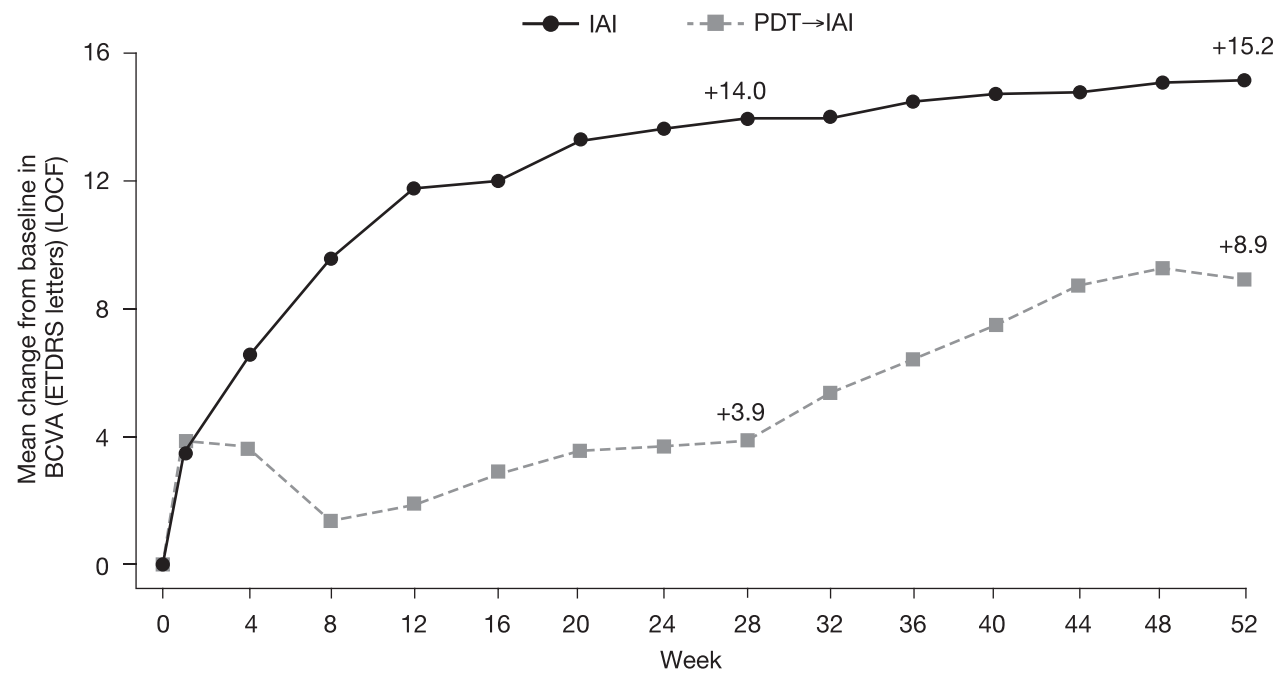

FIG. 2. Mean change in BCVA (letters) from baseline to week 52. BCVA improvements were maintained in the IAI group $(n=228)$ over the 52-week period, but there was further improvement after week 28 switch to IAI in the PDT $\rightarrow$ IAI group $(n=76)$. BCVA, best-corrected visual acuity; ETDRS, Early Treatment Diabetic Retinopathy Study; IAI, intravitreal aflibercept injections; LOCF, last observation carried forward; PDT, photodynamic therapy. difference (IAI-PDT $\rightarrow$ IAI) was +10.1 letters $(95 \%$ CI: 6.8 to $13.4 ; P<0.0001)$ at week 28 and +6.2 letters $(95 \% \mathrm{CI}: 2.6$ to $9.9 ; P=0.0009$ ) at week 52 (Table 2).

The proportion of patients maintaining vision (loss of $<15$ letters) was $98.7 \%(\mathrm{IAI})$ and $92.1 \%(\mathrm{PDT} \rightarrow \mathrm{IAI})$ at week 28 and $97.4 \%(\mathrm{IAI})$ and $90.8 \%(\mathrm{PDT} \rightarrow \mathrm{IAI})$ at week 52 , respectively. The LSM between-group difference was 6.6 (95\% CI: 0.4 to $12.9 ; P=0.0359$ ) at week 28 and 6.7 (95\% CI: -0.1 to $13.5 ; P=0.0523$ ) at week 52 . Significantly more patients treated with IAI gained letters at week 28 (all $P<0.0001$ for the comparison) and week 52 (all $P<0.05$ ) compared with PDT $\rightarrow$ IAI, and fewer patients treated with IAI lost letters at week $28(P<0.05)$ and week 52 (Fig. 3). In the PDT $\rightarrow$ IAI group, the percentages of patients who gained $\geq 5, \geq 10$, and $\geq 15$ letters from week 28 (after switch) to week 52 were $49.3 \%, 28.2 \%$, and $19.7 \%$, respectively.

The EQ-5D total scores at baseline were comparable between the IAI (0.93) and PDT (0.95) groups (Table 1). The mean change from baseline in the EQ-5D total score was
0.0175 for IAI and -0.0275 for PDT $\rightarrow$ IAI at week 28 . The improvement in EQ-5D total score was maintained at week 52 in the IAI group (0.0148). There was also a small improvement from weeks 28 to 52 (after switch) in the PDT $\rightarrow$ IAI group of 0.0134 , with a final mean change from baseline of -0.0144 . The LSM between-group difference in the EQ-5D total score favored IAI at week $28(0.029$ [95\% CI: 0.003 to 0.055 ]; $P=0.0284)$ and at week $52(0.0155$ [95\% CI: -0.0127 to 0.0437]; $P=0.2811)$. The NEI VFQ-25 total scores at baseline were also comparable between the IAI (74.1) and PDT (73.9) groups (Table 1). Clinically meaningful improvements ${ }^{12}$ in NEI VFQ-25 composite score were reported in the IAI and PDT $\rightarrow$ IAI treatment groups. The mean increase from baseline was 5.42 versus 2.68 at week 28 and 6.71 versus 6.03 at week 52. There was a further increase from week 28 to week 52 (after switch) in the PDT $\rightarrow$ IAI group of 3.45 . The LSM between-group difference was 2.81 (95\% CI: -0.40 to 6.02 ; $P=0.0860)$ at week 28 and $0.90(95 \% \mathrm{CI}:-2.21$ to 4.01 ; $P=0.5697)$ at week 52 .

Table 2. Least-Squares Mean Change from Baseline in Best-Corrected Visual Acuity (Letters) for Subgroups Treated With Intravitreal Aflibercept Injections $(N=228)$ or Photodynamic ThERAPY $\rightarrow$ INTRAVITREAL AFLIBERCEPT INJECTIONS $(N=76)$

\begin{tabular}{|c|c|c|c|c|c|c|c|c|}
\hline \multirow[b]{2}{*}{ Subgroup } & \multicolumn{4}{|c|}{ Baseline to week 28} & \multicolumn{4}{|c|}{ Baseline to week 52} \\
\hline & $\begin{array}{l}\text { IAI } \\
\text { group }\end{array}$ & $\begin{array}{l}P D T \rightarrow I A I \\
\text { group }\end{array}$ & $\begin{array}{c}\text { Difference } \\
(95 \% \text { CI })\end{array}$ & $\mathrm{P}$ & $\begin{array}{l}\text { IAI } \\
\text { group }\end{array}$ & $\begin{array}{l}P D T \rightarrow I A I \\
\text { group }\end{array}$ & $\begin{array}{c}\text { Difference } \\
(95 \% \text { CI })\end{array}$ & $\mathrm{P}$ \\
\hline Overall & +13.5 & +3.4 & $10.1(6.8$ to 13.4$)$ & $<0.0001$ & +14.2 & +7.9 & $+6.2(2.6$ to 9.9$)$ & 0.0009 \\
\hline Male & +13.4 & +2.8 & $10.6(6.5$ to 14.7$)$ & $<0.0001$ & +14.5 & +7.1 & $+7.4(2.7$ to 12.1$)$ & 0.0023 \\
\hline Female & +13.6 & +5.0 & $8.7(3.0$ to 14.3$)$ & 0.0029 & +13.1 & +10.3 & $+2.8(-3.1$ to 8.7$)$ & 0.3516 \\
\hline$<65$ years & +15.0 & +7.3 & $7.6(3.2$ to 12.1$)$ & 0.0008 & +15.4 & +12.5 & $+2.9(-1.7$ to 7.5$)$ & 0.2197 \\
\hline$\geq 65$ to $<75$ years & +12.7 & +0.3 & $12.4(7.1$ to 17.7$)$ & $<0.0001$ & +13.5 & +4.6 & $+8.9(2.6$ to 15.1$)$ & 0.0058 \\
\hline$\geq 75$ years & +11.6 & -3.8 & $15.5(5.6$ to 25.3$)$ & 0.0028 & +12.3 & -1.4 & $+13.7(2.4$ to 24.9$)$ & 0.0182 \\
\hline $\mathrm{CNV}<50 \%$ of lesion & +17.0 & +7.6 & $9.4(3.3$ to 15.4$)$ & 0.0027 & +18.1 & +12.7 & $+5.4(-0.9$ to 11.6$)$ & 0.0912 \\
\hline $\mathrm{CNV} \geq 50 \%$ of lesion & +11.3 & +0.4 & $10.9(7.1$ to 14.7$)$ & $<0.0001$ & +12.1 & +4.6 & $+7.5(3.0$ to 11.9$)$ & 0.0012 \\
\hline BCVA $<45$ letters & +15.5 & +7.4 & $8.0(1.4$ to 14.6$)$ & 0.0176 & +17.0 & +13.2 & $+3.9(-2.9$ to 10.6$)$ & 0.2610 \\
\hline $\mathrm{BCVA} \geq 45$ letters & +13.3 & +2.1 & $11.2(7.5$ to 14.9$)$ & $<0.0001$ & +14.2 & +6.8 & $+7.4(3.0$ to 11.8$)$ & 0.0010 \\
\hline
\end{tabular}

This highlights that IAI was effective in improving BCVA in youngest patients $(<65$ years) and in those with a smaller active component of the CNV lesion. Descriptive $P$-values based on treatment difference (IAI-PDT $\rightarrow$ IAI) of the LSM change using an analysis of covariance with treatment group and baseline BCVA group ( $<45, \geq 45$ letters) as fixed factors and baseline value as a covariate; evaluated using full analysis set/last observation carried forward approach.

BCVA, best-corrected visual acuity; CI, confidence interval; CNV, choroidal neovascularization; IAI, intravitreal aflibercept injections; LSM, least-squares mean; PDT, photodynamic therapy. 

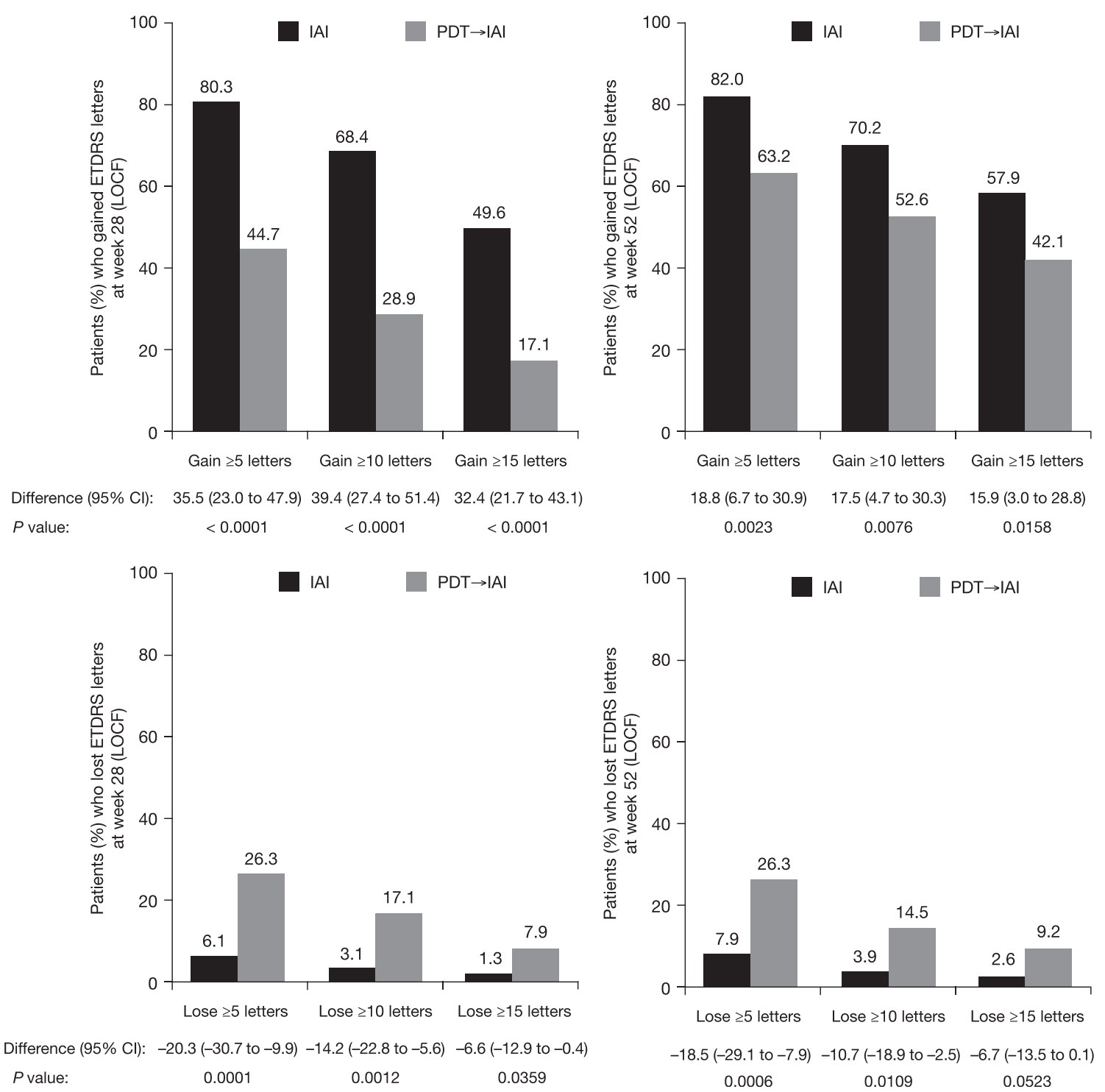

FIG. 3. Proportion of patients who gain or lose $\geq 5, \geq 10$, or $\geq 15$ letters from baseline to weeks 28 and 52 in the IAI $(n=228)$ and PDT $\rightarrow$ IAI $(n=76)$ treatment groups. Descriptive $P$-values based on treatment difference (IAI-PDT $\rightarrow$ IAI) using the Cochrane-Mantel-Haenszel test adjusted by baseline BCVA group; evaluated using full analysis set/LOCF approach. BCVA, best-corrected visual acuity; CI, confidence interval; ETDRS, Early Treatment Diabetic Retinopathy Study; IAI, intravitreal aflibercept injections; LOCF, last observation carried forward; PDT, photodynamic therapy.

\section{Morphologic outcomes}

The mean reduction in CRT from baseline to week 28 was $-180.8 \mu \mathrm{m}$ in the IAI group compared with $-98.2 \mu \mathrm{m}$ in the $\mathrm{PDT} \rightarrow \mathrm{IAI}$ group. The reduction in CRT was maintained at week 52 in the IAI group $(-189.6 \mu \mathrm{m})$, and there was a further reduction from weeks 28 to 52 (after switch) in the PDT $\rightarrow$ IAI group of $-76.9 \mu \mathrm{m}$, with a final mean change from baseline of $-170.0 \mu \mathrm{m}$ (Fig. 4A). From baseline to week 28, the LSM between-group difference was $-88.9 \mu \mathrm{m}(95 \% \mathrm{CI}:-112.3$ to $-65.5 ; P<0.0001)$ and from baseline to week 52, it was $-27.0 \mu \mathrm{m}(95 \% \mathrm{CI}:-45.9$ to $-8.2 ; P=0.0051)$ (Table 3$)$.

The mean reduction in CNV lesion size from baseline to week 28 in the IAI group $\left(-0.899 \mathrm{~mm}^{2}\right)$ was maintained at week 52 $\left(-1.007 \mathrm{~mm}^{2}\right)$ (Fig. 4B). A substantial mean reduction in CNV lesion size of $-0.867 \mathrm{~mm}^{2}$ was observed in the PDT $\rightarrow$ IAI group from baseline to week 52 compared with $-0.158 \mathrm{~mm}^{2}$ from baseline to week 28; there was a substantial improvement after switch to IAI at week 28 in this group of $-0.749 \mathrm{~mm}^{2}$. The LSM between-group difference was $-0.571 \mathrm{~mm}^{2}$ (95\% CI: -1.050 to $-0.092 ; P=0.0196)$ at week 28 , but was not statistically significant at week $52\left(-0.137 \mathrm{~mm}^{2}\right.$ [95\% CI: -0.580 to 0.307 ; $P=0.5445)$.

\section{Functional and morphologic outcomes in subgroups}

The 52-week results of prespecified subgroup analyses were consistent with the overall study results (Table 2). IAI was numerically greater than PDT $\rightarrow$ IAI in improving BCVA (letter score) across all subgroups, and these differences were more pronounced in male patients, those aged $\geq 65$ to $<75$ years and $\geq 75$ years, and in those with CNV $\geq 50 \%$ of total lesion. For both IAI and PDT $\rightarrow$ IAI, the greatest improvements in BCVA (letter score) were seen for patients in the youngest age group $(<65$ years), in those with 

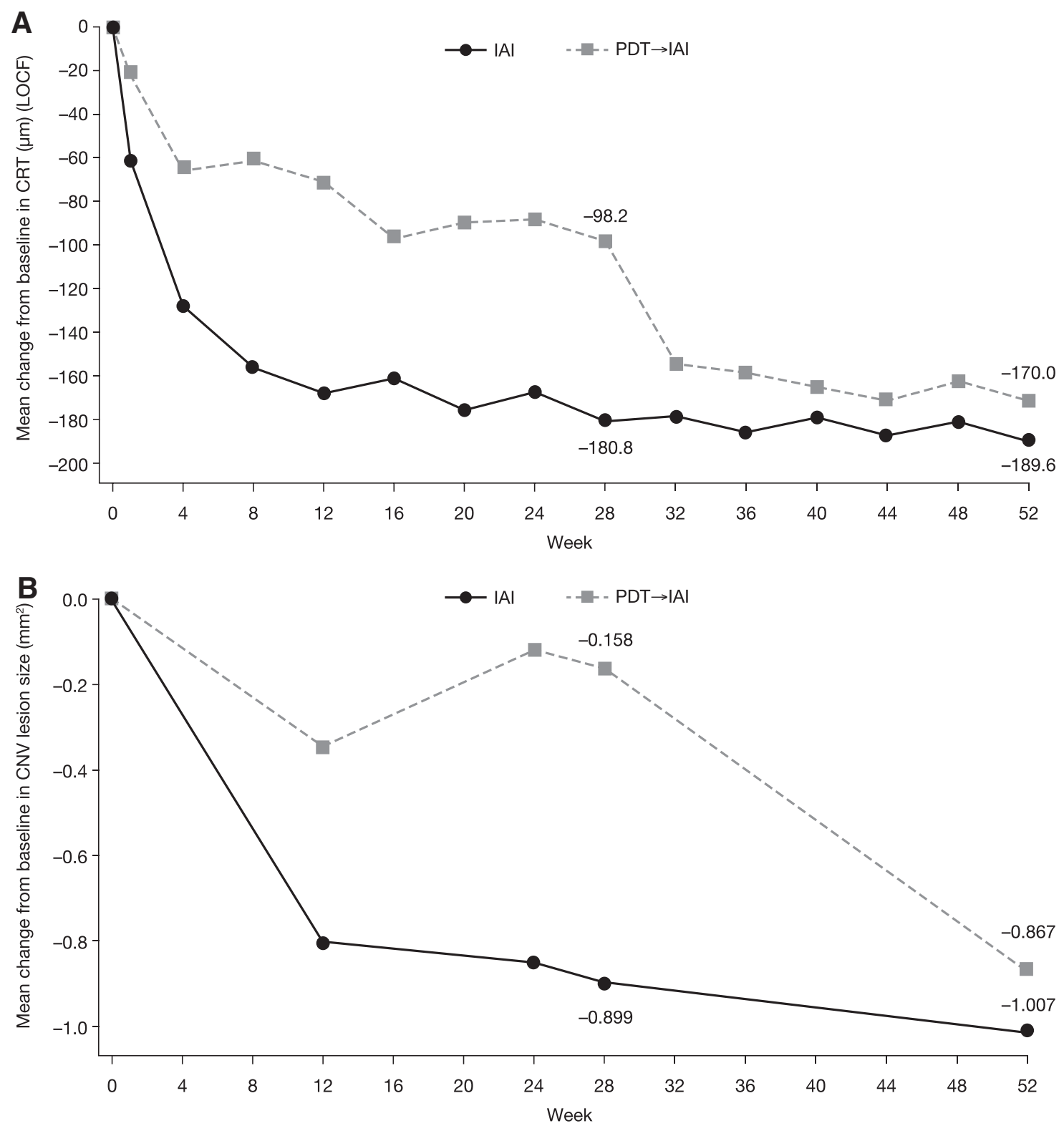

FIG. 4. Mean change in (A) CRT and (B) CNV lesion size from baseline. Morphologic benefits were maintained in the IAI group $(n=228)$ over the 52-week period, but there was further improvement after week 28 switch to IAI in the PDT $\rightarrow$ IAI group $(n=76)$. CNV, choroidal neovascularization; CRT, central retinal thickness; IAI, intravitreal aflibercept injections; PDT, photodynamic therapy.

a smaller active component of the CNV lesion $(<50 \%$ of lesion size), and with baseline BCVA $<45$ letters (Table 2). Similar between-group differences were observed with respect to CRT, but the greatest morphologic improvements with IAI were in patients with BCVA $<45$ letters (Table 3 ). It is interesting to note that the correlation coefficient between small active CNV lesions and total baseline lesion size was weak (0.15). This indicates that a greater treatment effect demonstrated in patients with a smaller active component of the CNV lesion was not associated with a smaller total lesion size at baseline.

\section{Safety outcomes}

Over the 52-week period, the incidence of TEAEs was similar in the IAI and PDT $\rightarrow$ IAI groups $(72.8 \%$ vs. $72.4 \%$, respectively) and the maximum intensity of $\mathrm{AE}$ for most patients with TEAEs was mild $(144 / 221 ; 65.2 \%)$ or moderate $(54 / 221 ; 24.4 \%)$ (Table 4$)$. The incidence of ocular TEAEs was similar in the IAI and PDT $\rightarrow$ IAI groups (51.8\% vs. $55.3 \%$, respectively). The most common ocular TEAEs were macular fibrosis, visual acuity reduced, and conjunctival hemorrhage. Intraocular pressure increased in $5.3 \%$ of patients in the IAI group; however, all patients recovered and there was no drug interruption or withdrawal. There were no reports of endophthalmitis.

For patients in the PDT $\rightarrow$ IAI group, no new safety signal was observed in the second half of the year (from weeks 28 to 52) after switching to IAI at week 28 (Supplementary Table S2). Only one serious ocular TEAE (cataract) in the PDT $\rightarrow$ IAI group was considered drug related, and it occurred after switch to IAI at week 28. 


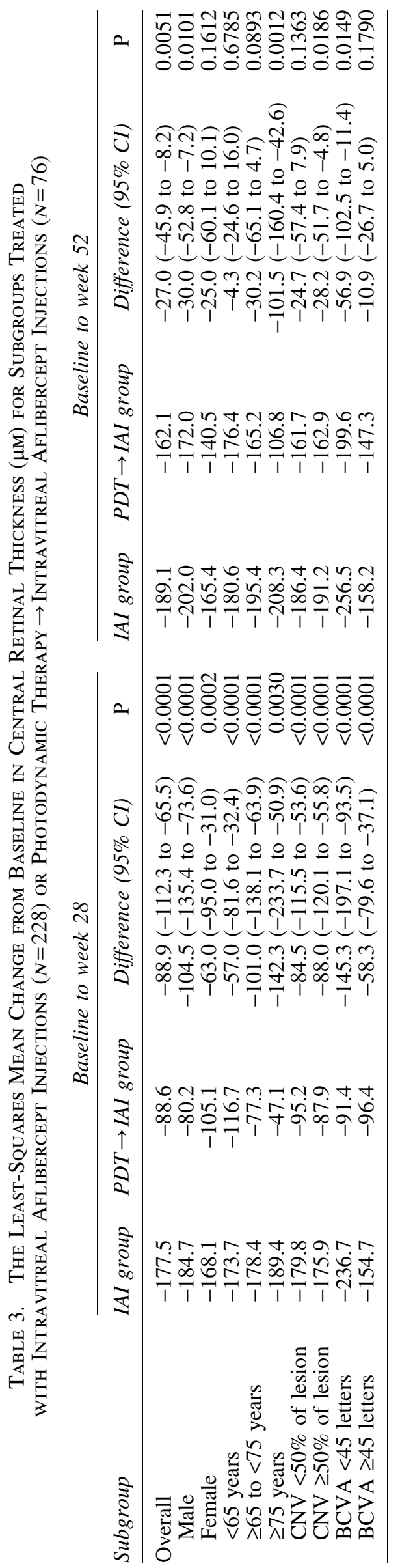

Table 4. Number (\%) of Patients With TREATMENT-EMERgENT AdVERSE Events IN EITHER TREATMENT GROUP REPORTED FROM BASELINE TO WEEK 52

\begin{tabular}{|c|c|c|}
\hline n (\%), safety analysis set & $\begin{array}{c}I A I \\
(\mathrm{n}=228)\end{array}$ & $\begin{array}{l}P D T \rightarrow I A I \\
(\mathrm{n}=76)\end{array}$ \\
\hline Any TEAE & $166(72.8)$ & $55(72.4)$ \\
\hline Mild & $112(49.1)$ & $32(42.1)$ \\
\hline Moderate & 37 (16.2) & $17(22.4)$ \\
\hline Severe & $17(7.5)$ & $6(7.9)$ \\
\hline Any serious TEAEs & $18(7.9)$ & $7(9.2)$ \\
\hline Any ocular TEAE (study eye) & $118(51.8)$ & $42(55.3)$ \\
\hline \multicolumn{3}{|c|}{$\begin{array}{l}\text { Ocular TEAE (study eye) with incidence } \\
\geq 5 \% \text { at week } 52^{\mathrm{a}}\end{array}$} \\
\hline Conjunctival hemorrhage & $20(8.8)$ & $6(7.9)$ \\
\hline Intraocular pressure increased & $12(5.3)$ & $1(1.3)$ \\
\hline Macular degeneration & $9(3.9)$ & $4(5.3)$ \\
\hline Macular fibrosis & $27(11.8)$ & $5(6.6)$ \\
\hline Macular edema & $4(1.8)$ & $5(6.6)$ \\
\hline Retinal hemorrhage & $5(2.2)$ & $4(5.3)$ \\
\hline Retinal pigment epitheliopathy & $12(5.3)$ & $7(9.2)$ \\
\hline Subretinal fluid & $3(1.3)$ & $5(6.6)$ \\
\hline Visual acuity reduced & $15(6.6)$ & $16(21.1)$ \\
\hline Visual acuity tests abnormal & $3(1.3)$ & $4(5.3)$ \\
\hline $\begin{array}{l}\text { Any ocular serious TEAE } \\
\text { (study eye) })^{\mathrm{a}}\end{array}$ & $1(0.4)$ & $4(5.3)$ \\
\hline Cataract & 0 & $1(1.3)$ \\
\hline Visual acuity reduced & 0 & $3(3.9)$ \\
\hline Vitreous hemorrhage & $1(0.4)$ & 0 \\
\hline Any nonocular TEAE & $114(50.0)$ & $33(43.4)$ \\
\hline Any nonocular serious TEAE & $15(6.6)$ & $3(3.9)$ \\
\hline Death ${ }^{b}$ & $1(0.4)$ & 0 \\
\hline APTC-ATE & $1(0.4)$ & $2(2.6)$ \\
\hline Nonfatal $\mathrm{MI}^{\mathrm{a}}$ & 0 & $1(1.3)$ \\
\hline Nonfatal stroke $^{\mathrm{a}}$ & $1(0.4)$ & $1(1.3)$ \\
\hline
\end{tabular}

${ }^{\mathrm{a}}$ MedDRA version 17.0 preferred terms.

${ }^{\mathrm{b}}$ Not treatment emergent, not considered drug related.

APTC-ATE, Antiplatelet Trialists' Collaboration-defined arterial thromboembolic event; IAI, intravitreal aflibercept injections; MI, myocardial infarction; PDT, photodynamic therapy; TEAE, treatmentemergent adverse event.

Three APTC-ATE events (one acute myocardial infarction in the category of nonfatal myocardial infarction; two cerebral infarctions in the category of nonfatal stroke) were observed; these were not considered related to the study drug. There was one death related to hepatocellular carcinoma in the IAI group that was not considered study drug related. Only one treatment-emergent persistent positive antibody occurred in the IAI group at week 52 (visit 16). Prespecified ocular and nonocular TEAEs of interest are reported in Supplementary Tables 3 and 4.

\section{Discussion}

This article describes the final results from SIGHT, the first randomized study to compare IAI with PDT $\rightarrow$ IAI in Chinese patients with predominantly classic CNV secondary to nAMD. Consistent with previous anecdotal reports on PDT in Asian populations, ${ }^{5,6}$ quite favorable outcomes with a gain of almost one line of vision were observed with PDT treatment up to week 28. Despite the good performance of PDT, IAI $(2 \mathrm{mg}$ every 8 weeks after 3 initial monthly 
injections) was still clinically and statistically superior to PDT with an average gain in visual acuity of $\sim 3$ lines. After switching the PDT-treated patients to IAI at week 28 , there was a further improvement in visual acuity; however, the outcome at week 52 was still inferior to patients receiving IAI from the study start. This was also reflected in visionrelated quality-of-life outcomes; IAI was associated with an early improvement in both EQ-5D and NEI-VFQ-25 scores, which were maintained during the study. Switching PDT treated to IAI was associated with an improvement in these measures. Hence, IAI therapy initiated early in the course of the disease is superior over PDT even if patients can be switched to IAI later.

The visual acuity outcomes observed with PDT treatment in SIGHT were better than those reported in previous randomized controlled studies such as ANCHOR and TAP. ${ }^{2,4}$ In SIGHT, $90.8 \%$ of patients in the PDT group maintained vision at week 52. The corresponding numbers in ANCHOR and TAP were $64.3 \%$ and $61 \%$, respectively; it must also be noted that mean CNV lesion size increased by $1.63 \mathrm{~mm}^{2}$ over 52 weeks in ANCHOR. ${ }^{2,4}$ In SIGHT, there was a mean decrease in CNV lesion size of $1.0 \mathrm{~mm}^{2}$ at week 52 . Since ANCHOR and TAP primarily included white patients, the question arises whether the better outcomes in SIGHT were attributable to ethnic differences as implied earlier by anecdotal reports, suggesting that PDT results are better in Asian patients. ${ }^{5,6}$ To address this question, a systematic analysis of disease characteristics and efficacy subgroup analyses was prespecified in SIGHT.

Based on these analyses, it is important to note that the Chinese nAMD patients in SIGHT had disease features that differed from those of the patients enrolled in ANCHOR. Patients in SIGHT were younger (mean age: 65 years), had a smaller total lesion size $\left(3.26 \mathrm{~mm}^{2}\right)$ and $\mathrm{CNV}$ lesion size $\left(1.83 \mathrm{~mm}^{2}\right)$, which may have resulted in better outcomes with IAI over PDT. In VIEW 1 and 2, 23.6\%-28.8\% of patients had predominantly classic $\mathrm{CNV}$, the mean age was 73.8-77.9 years, the mean total lesion size was 6.89$8.22 \mathrm{~mm}^{2}$, the mean $\mathrm{CNV}$ area was $6.57-7.75 \mathrm{~mm}^{2}$, and the mean CRT was 324.4-342.6 $\mu \mathrm{m}$ at baseline (across IAI 2q8 groups). ${ }^{7,8}$ The mean CRT at baseline was $460 \mu \mathrm{m}$ in SIGHT. In ANCHOR, most patients were white (98\%) with predominantly classic CNV $(97 \%)$, but the mean age was higher (77 years) than in SIGHT. ${ }^{2}$ There was also a difference between ANCHOR and SIGHT in the proportion of male patients $(64.5 \%$ vs. $50.1 \%){ }^{2}$ Subgroup analyses showed that these factors, in particular, drove the more positive outcomes of the PDT group in SIGHT than in the historic studies, while results are consistent across studies for patients with similar baseline characteristics. ${ }^{13,14}$

With regard to the IAI-treated patients, the findings from SIGHT are consistent with those reported in VIEW. ${ }^{7,8} \mathrm{~A}$ subanalysis of patients with predominantly classic CNV who were randomized to IAI $2 \mathrm{mg}$ every 8 weeks after 3 initial monthly doses in VIEW (data on file) showed that the mean change in BCVA was +9.4 letters in white $(n=135)$ and +11.4 letters in Asian $(n=18)$ patients at week 28, and the proportions who lost $<15$ letters at week 28 were $97.8 \%$ and $100.0 \%$, respectively. The mean change in CRT was $-150.4 \mu \mathrm{m}$ (white) and $-184.9 \mu \mathrm{m}$ (Asian) at week 28. In PDT-treated patients, the slightly better outcomes for mean BCVA change in the SIGHT study may potentially be associated with the lower mean age (65 years) compared with the overall VIEW study population (76 years [white] and 69 years [Asian]) (data on file).

SIGHT also demonstrated the benefits of IAI in patients who switched from PDT to IAI at week 28. However, the benefits were generally lower than in patients who initially started with IAI. In addition, it is possible that laser scarring and other PDT-related side effects (such as defects in the visual field), as well as disease progression despite PDT, could lead to ceiling effects, which may have contributed to the inferior outcomes in the PDT group at week 52. The findings in younger patients and in those with a smaller size of the active CNV lesion may further support the benefit of early treatment initiation with IAI. Of note, the size of the active CNV lesion is an independent factor from total lesion size as the correlation was weak (correlation coefficient $0.15)$.

The safety profile of IAI in Asian patients was, in general, comparable with that observed in the VIEW studies; the incidence of serious ocular TEAEs from baseline to week 52 was $0.4 \%$ in the IAI group compared with $2 \%$ in the IAI $2 q 8$ group in VIEW combined. ${ }^{7,8}$ The higher reporting of macular fibrosis in the IAI group of SIGHT is most likely attributable to improved visibility of anatomic details after clearing of the retinal bleeds and edema after the first IAI. In contrast, PDT alters macular morphology and may induce some scaring, which further interferes with the visibility of pathologic-anatomic details resulting in a higher incidence of visual acuity-reduced side effects.

It should be noted that the study has a number of limitations. PDT was used according to label, meaning that the study was focused on predominantly classic CNV patients only. Other key subgroups that are relevant to Asian populations, such as PCV, were excluded. Patients in the PDT group also switched to IAI at week 28 (after primary outcome measurement), which may have resulted in some confounding regarding 52-week comparisons.

In conclusion, IAI $2 q 8$ demonstrated significant superiority over PDT in Chinese patients with nAMD over a 52week period; these benefits were evident in a population where PDT was more effective than demonstrated in other studies. The 52-week incidence of AEs was consistent with the known safety profile of IAI. Importantly, it is clear that the efficacy and safety of IAI are consistent across patient population ethnicity in accordance with the International Conference for Harmonisation E5 guidance. ${ }^{15}$ These findings should help inform clinical decision-making with regard to treatment with IAI in Asian and in particular Chinese nAMD patients.

\section{Acknowledgments}

The following investigators were members of the SIGHT study group in China: Xiaoxin Li, People's Eye Center, Peking University People's Hospital, Beijing, China. Youxin Chen, Peking Union Medical Colleague Hospital, Beijing, China. Junjun Zhang, West China School of Medicine/ West China Hospital, Sichuan University, Chengdu, China. Xun Xu, Shanghai General Hospital, Shanghai, China. Feng Zhang, Beijing Tongren Hospital, Beijing, China. Zhizhong Ma, Peking University Third Hospital, Beijing, China. Gezhi Xu, Eye and ENT Hospital of Fudan University, Shanghai, China. Xiaoling Liu, The Affiliated Eye Hospital of Wenzhou Medical College, Zhejiang, China. Changxian 
Yi, Zhongshan Ophthalmic Center, Guangdong, China. Yusheng Wang, Xijing Hospital, Xi'an, China. Ke Yao, Zhejiang, China. Luosheng Tang, Hunan, China. Haifeng Xu, Qingdao Eye Hospital, Qingdao, China. Xiaorong Li, Tianjin, China. Medical writing assistance was provided by PAREXEL and was funded by Bayer Pharmaceuticals. The study was funded by Bayer Pharmaceuticals. The sponsor or funding organization participated in the design and conduct of the study, data collection, data management, data analysis, interpretation of the data, and preparation of article.

\section{Author Disclosure Statement}

X.L.: consultant/advisor (Bayer); research funding (Bayer). Y.C.: consultant/advisor (Bayer); research funding (Bayer). J.Z.: consultant/advisor (Bayer); research funding (Bayer). X.X.: consultant/advisor (Bayer); research funding (Bayer). C.M.G.C.: consultant/advisor (Bayer, Novartis); research funding (Bayer, Novartis, Roche); reimbursement (Bayer, Novartis, Allergan). R.Y.: employee (Bayer). H.K.: employee (Regeneron). O.S.: employee (Bayer). O.Z.: employee (Bayer).

\section{References}

1. Wong, W.L., Su, X., Li, X., et al. Global prevalence of agerelated macular degeneration and disease burden projection for 2020 and 2040: a systematic review and meta-analysis. Lancet Glob. Health. 2:e106-e116, 2014.

2. Brown, D.M., Kaiser, P.K., Michels, M., et al. Ranibizumab versus verteporfin for neovascular age-related macular degeneration. N. Engl. J Med. 355:1432-1444, 2006.

3. Brown, D.M., Michels, M., Kaiser, P.K., Heier, J.S., Sy, J.P., and Ianchulev, T. Ranibizumab versus verteporfin photodynamic therapy for neovascular age-related macular degeneration: two-year results of the ANCHOR study. Ophthalmology. 116:57-65, 2009.

4. Treatment of age-related macular degeneration with photodynamic therapy (TAP) Study Group. Photodynamic therapy of subfoveal choroidal neovascularization in age-related macular degeneration with verteporfin: one-year results of 2 randomized clinical trials-TAP report. Arch. Ophthalmol. 117:1329-1345, 1999.

5. Chen, Y.X., Ge, J., Yan, M., et al. [Clinical trial of verteporfin photodynamic therapy in Chinese age-related macular degeneration with subfoveal predominant choroidal neovascularization patients]. Zhonghua. Yan. Ke. Za. Zhi. 43: 198-205, 2007.

6. Yoon, J.U., Byun, Y.J., and Koh, H.J. Intravitreal antiVEGF versus photodynamic therapy with verteporfin for treatment of myopic choroidal neovascularization. Retina. 30:418-424, 2010.
7. Heier, J.S., Brown, D.M., Chong, V., et al. Intravitreal aflibercept (VEGF trap-eye) in wet age-related macular degeneration. Ophthalmology. 119:2537-2548, 2012.

8. Schmidt-Erfurth, U., Kaiser, P.K., Korobelnik, J.F., et al. Intravitreal aflibercept injection for neovascular age-related macular degeneration: ninety-six-week results of the VIEW studies. Ophthalmology. 121:193-201, 2014.

9. Wong, T.Y., Ogura Y. 2-Year (96-week) efficacy and safety of intravitreal aflibercept injection for neovascular age-related macular degeneration in Asian patients: subgroup analysis of VIEW studies. Abstract FP-FR-17-8. World Ophthalmology Congress, Tokyo, Japan, 2-6 April, 2014.

10. Sivaprasad, S., Saleh, G.M., and Jackson, H. Does lesion size determine the success rate of photodynamic therapy for agerelated macular degeneration? Eye (Lond.). 20:43-45, 2006.

11. Verteporfin Roundtable Participants. Guidelines for using verteporfin (Visudyne) in photodynamic therapy for choroidal neovascularization due to age-related macular degeneration and other causes: update. Retina. 25:119-134, 2005.

12. Suner, I.J., Kokame, G.T., Yu, E., Ward, J., Dolan, C., and Bressler, N. Responsiveness of NEI VFQ-25 to changes in visual acuity in neovascular AMD: validation studies from two phase 3 clinical trials. Invest. Ophthalmol. Vis. Sci. 50: 3629-3635, 2009.

13. Brown, D.M., Shapiro, H., and Schneider, S. Subgroup analysis of first-year results of ANCHOR: a phase III, double-masked, randomized comparison of ranibizumab and verteporfin photodynamic therapy for predominantly classic choroidal neovascularization related to age-related macular degeneration. Invest. Ophthalmol. Vis. Sci. 47: 2963, 2006.

14. Kaiser, P.K., Brown, D.M., Zhang, K., et al. Ranibizumab for predominantly classic neovascular age-related macular degeneration: subgroup analysis of first-year ANCHOR results. Am. J. Ophthalmol. 144:850-857, 2007.

15. ICH Harmonised Tripartite Guideline. Ethnic factors in the acceptability of foreign clinical data $\mathrm{EH}(\mathrm{R} 1)$. Available at www.ich.org/products/guidelines/efficacy/article/efficacyguidelines.html (accessed April 18, 2016).

Received: May 18, 2016 Accepted: December 15, 2016

Address correspondence to: Dr. Xiaoxin $\mathrm{Li}$

Department of Ophthalmology, People's Eye Center Peking University People's Hospital No. 11, Xizhimen South Street Xicheng District, Beijing, 100044 China

E-mail: dr_lixiaoxin@163.com 